\title{
High and low reactivity to novelty and sexual behavior in male rats
}

\author{
VALERIE LEMAIRE and ANDERS ÅGMO \\ University of Tours, Tours, France
}

\begin{abstract}
A rat's level of activity when exposed to a novel environment has been shown to be associated with enhanced sensitivity to the locomotor-activating and rewarding effects of dopamine-releasing substances. Male rats were divided into high responders (HRs) and low responders (LRs), depending on their activity in a 90-min test in an unknown environment. Tests for sexual behavior were then performed. It was found that the HRs had longer intromission and ejaculation latencies as well as reduced intromission ratios and number of ejaculations at the first test. The number of mounts made by the HRs, however, was higher than that made by the LRs. In the second and third tests, there was no difference between groups. Other groups of HRs and LRs were treated with amphetamine, $1 \mathrm{mg} / \mathrm{kg}$, before the sexual behavior test as well as before a second test of ambulatory activity. The locomotor response to amphetamine was greater for HRs than for LRs. However, with regard to sexual behavior, HRs differed from LRs in a way similar to that observed in untreated animals. These data show that there is no relationship between reaction to novelty and sexual motivation, and that the effects of amphetamine on sexual behavior are similar in HRs and LRs.
\end{abstract}

Rats exposed to a novel environment display a series of behaviors that are not observed, or that are much reduced, in animals familiarized with the environment (Berlyne, Koenig, \& Hirota, 1966). The critical role of accumbens dopamine in the behavioral activation produced by novelty has been established in pharmacological studies (Hooks \& Kalivas, 1995; Pierce, Crawford, Nonneman, Mattingly, \& Bardo, 1990). Rats that show high ambulatory activity in response to novelty, called high responders (HRs), have larger basal dopamine release in the nucleus accumbens than do rats that display low activity, or low responders (LRs) (Bradberry, Gruen, Berridge, \& Roth, 1991; Hooks, Colvin, Juncos, \& Justice, 1992). HRs also manifest a greater increase in dopamine release in the accumbens in response to amphetamine and cocaine (Hooks, Jones, Smith, Neill, \& Justice, 1991) and a greater increase in ambulatory activity after administration of these drugs than that observed in LRs (Exner \& Clark, 1993; Hooks \& Kalivas, 1994; Hooks et al., 1991). Furthermore, HRs are more sensitive to the reinforcing actions of intravenous amphetamine than are LRs, according to self-administration studies (Piazza, Deminière, Maccari, Le Moal, \& Simon, 1989; Piazza et al., 1990; Piazza, Maccari, et al., 1991). These observations suggest that HRs not only release more dopamine in response to novelty than do LRs, but also that their responses to this transmitter are enhanced. Moreover, animals characterized as high feeders (on the basis of sucrose intake) show a

\footnotetext{
The authors thank Ann Marie Le Guisquet and Serge Barreau for assistance with the data collection. Correspondence should be addressed to A. Ågmo, Laboratoire de Psychophysiologie, Faculté des Sciences, Université de Tours, Parc de Grandmont, 37200 Tours, France (e-mail: agmo@balzac.univ-tours.fr).
}

greater locomotor response to amphetamine than do low feeders (Sills \& Vaccarino, 1994). This observation indicates that there is an overlap in the mechanisms that determine the reward value of food, reactivity to novelty, and sensitivity to amphetamine.

Dopamine-releasing drugs and dopamine-receptor agonists are known to facilitate the initiation of sexual behavior in male rats without sexual experience ( $\AA$ gmo \& Picker, 1990) and in male rats where this behavior is of low intensity, such as castrated ( $\AA$ gmo \& Fernández, 1989; Malmnäs, 1977; Scaletta \& Hull, 1990) or frontalcortex-lesioned animals (Ågmo \& Villalpando, 1995). Interestingly, facilitated sexual behavior is associated with enhanced exploratory activity in a novel environment ( $\AA$ gmo \& Picker, 1990). Furthermore, dopaminergic stimulants facilitate instrumental responding for sexual reward and reduce mount latency after injection directly into the nucleus accumbens (Everitt, 1990). Microdialysis and voltammetric studies have shown that the exposition to sexually relevant stimuli, such as the odor or sight of a receptive female, enhances dopamine release in this nucleus (Damsma, Pfaus, Wenkstern, Phillips, \& Fibiger, 1992; Louilot, Gonzalez-Mora, Guadalupe, \& Mas, 1991; Mitchell \& Gratton, 1991; Pfaus et al., 1990). There is, then, a considerable amount of evidence showing that the initiation of sexual behavior is preceded by enhanced dopamine release in the accumbens. The physiological importance of this release is substantiated by a report suggesting that inexperienced male rats that do not copulate when exposed to a receptive female release less dopamine in the nucleus accumbens than those that do copulate (Wenkstern, Pfaus, \& Fibiger, 1993). Moreover, dopamine release is significantly lower in castrated animals that do not copulate than it is in those that do copulate (Hull, Du, Lorrain, \& Matuszewich, 1995). 
Since HRs present a larger dopamine release in the nucleus accumbens than do LRs in response to novelty, it could be predicted that the former would display higher sexual motivation than the latter at their first encounter with a receptive female in an unknown environment. Furthermore, since dopamine is of slight importance both for the initiation of sexual behavior in sexually experienced rats and for the execution of this behavior once initiated ( gmo \& Fernández, 1989; Ågmo \& Villalpando, 1995), the difference between HRs and LRs should disappear upon repeated testing and always be limited to parameters of sexual motivation. Furthermore, the facilitatory effects of amphetamine should be greater in HRs than in LRs. These predictions were tested in the following experiments.

\section{METHOD}

\section{Subjects}

Wistar rats (males weighing 350-400 g, females weighing about $250 \mathrm{~g}$ ) from Iffa Credo (L'Arbresle, France) were housed under a reversed light:dark cycle $(12: 12 \mathrm{~h}$, lights on $2000 \mathrm{~h})$ at an ambient temperature of $22^{\circ} \pm 2^{\circ} \mathrm{C}$ with commercial rat pellets and water available ad lib. Males and females were kept in the same room in Macrolon cages $(24 \times 36 \mathrm{~cm})$ with 2 or 3 animals per cage. The females were ovariectomized under methohexital (Lilly) anesthesia $(40 \mathrm{mg} / \mathrm{kg})$ at least 2 weeks before being used. They were given a sequential treatment with estradiol benzoate $(25 \mu \mathrm{g} / \mathrm{rat})$ and progesterone ( $2 \mathrm{mg} / \mathrm{rat}$ ) (about 48 and $4 \mathrm{~h}$ before test, respectively) to induce intense proceptive and receptive behaviors. Both steroids were purchased from Sigma, dissolved in peanut oil, and injected subcutaneously in a volume of $0.2 \mathrm{ml}$.

\section{Procedure}

Ambulatory activity test. About 3 weeks after arrival in the laboratory, the males were individually placed in a novel environment consisting of a circular steel corridor $(10 \mathrm{~cm}$ wide and with walls $70 \mathrm{~cm}$ high and an inner diameter of $55 \mathrm{~cm}$ ) with infrared photocells placed $2.5 \mathrm{~cm}$ above the grid floor at each cardinal point. A beam interruption of $250 \mathrm{msec}$ was required to activate the counters. In that way, rapid movements were not registered. The activity count represents, therefore, basically ambulatory activity. The number of photocell interruptions was registered each $15 \mathrm{~min}$ during the 90 min following introduction of the animal to the apparatus. This apparatus is similar to those used in other studies of reactivity to novelty (Deroche, Piazza, Le Moal, \& Simon, 1993).

Tests for sexual behavior. A cylindrical Plexiglas arena (diameter $60 \mathrm{~cm}$ ) was used with the floor covered by sawdust. This arena was placed on a table in the middle of a large room, allowing for exposure to visual stimuli. This assured novelty. The male was introduced into the arena and $10 \mathrm{~min}$ later a receptive female was introduced. The following parameters of sexual behavior were registered: mount and intromission latency (time from introduction of the female until the first mount and intromission, respectively), ejaculation latency (time from the first intromission until ejaculation), postejaculatory interval (time from ejaculation until the next intromission), and number of mounts and number of intromissions. The intromission ratio (the number of intromissions divided by the number of mounts plus intromissions) was also calculated for animals making at least one mount. The test lasted 45 min after introduction of the female. On subsequent mating tests, the male was placed in the arena for $5 \mathrm{~min}$ before introduction of the female. The tests were ended at the end of the first postejaculatory interval, or when mount, intromission, or ejaculation latency exceeded $30 \mathrm{~min}$.

\section{Design}

When the rats had been tested for ambulatory activity, they were separated into two groups. The rats with a total activity score above the median formed the HR group $(N=15)$; the rats with an activity score below the median formed the LR group $(N=16)$. This is the traditional way of obtaining HR and LR rats (Deroche et al., 1993; Erb \& Parker, 1994; Hooks \& Kalivas, 1994; Hooks et al., 1991). About 1 week after the test for ambulatory activity, the sexual behavior tests were initiated. They were then performed once a week for 3 weeks. In a second experiment, males were identified as HR $(N=8)$ or LR $(N=8)$ as described above. Forty minutes before the only sexual behavior test, they were intraperitoneally injected with $1 \mathrm{mg} / \mathrm{kg}$ of $d$-amphetamine sulfate (Sigma) dissolved in physiological saline. The injection volume was $1 \mathrm{ml} / \mathrm{kg}$ body weight. The test was ended $30 \mathrm{~min}$ after introduction of the female. About 1 week later, the animals were again injected with $d$-amphetamine, $1 \mathrm{mg} / \mathrm{kg}$, and ambulatory activity was recorded as described above for $90 \mathrm{~min}$ following injection.

\section{RESULTS}

The activity score in the different groups is shown in Figure 1. No statistical comparison was made between untreated LRs and HRs because these groups were obtained a posteriori by separating the animals above the median from those below. However, activity data after $d$-amphetamine treatment was evaluated by an analysis of covariance with the activity at the first test (B1 in Figure 1) used as covariate. This eliminated the influence of initial differences in ambulatory activity. Data were transformed into square roots before analysis in order to homogenize error variances. A significant group difference was obtained $[F(1,13)=5.36, p<.05]$. This shows that HRs were indeed more sensitive to amphetamine than were LRs.

Analyses of sexual behavior data from the first experiment showed that the groups differed only at the first

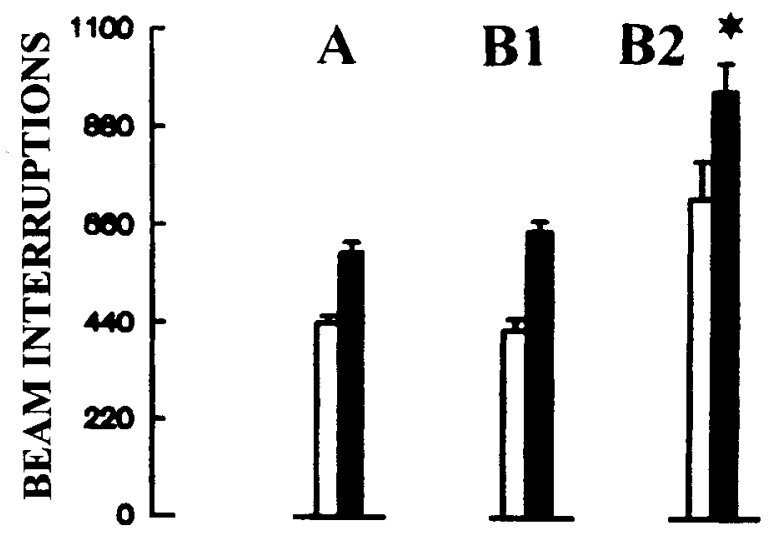

Figure 1. Total activity in the 90-min test. A, first exposure to the apparatus in animals that were tested for sexual behavior without drug treatment. B1, first exposure to the apparatus in animals that were tested for sexual behavior after treatment with $d$-amphetamine, $1 \mathrm{mg} / \mathrm{kg}$. B2, data from the latter animals at a second test in which $d$-amphetamine, $1 \mathrm{mg} / \mathrm{kg}$, was injected $5 \mathrm{~min}$ before testing. ${ }^{*} p<.05$. Open bars, LRs; black bars, HRs. 
test. Subsequent tests are, therefore, not further discussed and data are not shown. The proportion of animals displaying mounts, intromissions, and ejaculation were similar in the HR and LR groups, as evaluated by the chisquare test. The apparent difference in ejaculation percentage failed to reach statistical significance. There was no difference in mount latency, but the intromission and ejaculation latencies were longer in the HRs than in the LRs. No effect was found on the postejaculatory interval (data not shown). The number of mounts was greater in the HRs, but there was no difference between the groups with regard to the number of intromissions. The number of ejaculations achieved during the test was lower in the HRs than in the LRs. It must be remembered that the first test lasted $45 \mathrm{~min}$, and that the rats were allowed to make multiple ejaculations. Finally, the intromission ratio was lower in the HRs. The data are summarized in Table 1.

In the second experiment, that is, after treatment with amphetamine at a dose of $1 \mathrm{mg} / \mathrm{kg}$, HRs again displayed a larger number of mounts than did LRs and had longer ejaculation latencies and lower intromission ratios. Thus, the groups differed in a way similar to that observed in the untreated animals. The only group difference that was not replicated was the longer intromission latency observed in the HRs in the first experiment.

\section{DISCUSSION}

Sexual motivation (libido), defined as the ease with which sexual behavior is activated, is frequently distinguished from the execution of copulatory acts (potency) (Clark, 1993; Davidson, 1980). The number of mounts and the mount latencies are believed to reflect motivation, while the number of intromissions and the intromission ratio are indicators of copulatory efficiency (Clark, 1993). The larger number of mounts made by the HRs in both experiments could suggest that these animals were more sexually motivated than the LRs. However, another indicator of sexual motivation, the mount latency, was not significantly different between the two groups. It is possible that a factor other than enhanced sexual motivation may have caused the increased number of mounts. An important observation in this context is that the intromission ratio was lower for the HRs than for the LRs. This suggests that the HRs had some difficulty in achieving vaginal penetration. Treatments that interfere with intromission, such as section of the dorsal penile nerve, anesthesia of the glans penis, or some pharmacological agents, increase the number of mounts (Contreras \& Agmo, 1993; Meisel \& Sachs, 1994; Paredes, Holmes, Sachs, \& Agmo, 1993). There is no evidence that these treatments enhance sexual motivation. Thus, the increased number of mounts in the HR rats may be secondary to the reduced intromission ratio. This suggestion is supported by the data showing increased intromission and ejaculation latencies in the HRs, something that does not coincide with enhanced sexual motivation but is concordant with deficient intromission behavior. The longer ejaculation latency in the HRs could also explain the lower number of ejaculations performed by these animals in the first experiment. No difference in this number was obtained in the second experiment, but this may be due to the shorter duration of the test.

It is possible that measures of sexual motivation different from those used here would have given other results. However, the effects of dopaminergic stimulation on mount and intromission latencies are very similar to those obtained on leverpressing for access to a receptive female (Ågmo \& Picker, 1990; Everitt, 1990). Furthermore, dopamine antagonists fail to affect mount and intromission latencies in sexually inexperienced rats $(\AA g$ gmo \& Picker, 1990) and does not affect parameters of sexual motivation in an X-maze (Hull et al., 1991). It seems, therefore, that alterations in dopaminergic neurotransmission affect mount and intromission latencies in the same way that they affect other measures of sexual motivation. This makes it unlikely that HRs and LRs would have behaved differently in procedures specially designed for evaluation of sexual motivation. Nevertheless, this possibility cannot be excluded.

Table 1

Parameters of Sexual Behavior in Male Rats Classified as Low Responders (LRs) or High Responders (HRs) to Novelty Either Left Untreated Before the Sexual Behavior Test or Given an Injection of Amphetamine

\begin{tabular}{|c|c|c|c|c|c|c|c|c|}
\hline \multirow[b]{3}{*}{ Behavior Parameter } & \multicolumn{4}{|c|}{ Untreated } & \multicolumn{4}{|c|}{ Amphetamine $1 \mathrm{mg} / \mathrm{kg}$} \\
\hline & \multicolumn{2}{|c|}{ LRs } & \multicolumn{2}{|c|}{ HRs } & \multicolumn{2}{|c|}{ LRs } & \multicolumn{2}{|c|}{ HRs } \\
\hline & $M$ & $S E$ & $M$ & $S E$ & $M$ & $S E$ & $M$ & $\overline{S E}$ \\
\hline Mount & \multicolumn{2}{|c|}{$87 \%$} & \multicolumn{2}{|c|}{$93 \%$} & \multicolumn{2}{|c|}{$87 \%$} & \multicolumn{2}{|c|}{$75 \%$} \\
\hline Intromission & \multicolumn{2}{|c|}{$81 \%$} & \multicolumn{2}{|c|}{$73 \%$} & \multicolumn{2}{|c|}{$87 \%$} & \multicolumn{2}{|c|}{$62 \%$} \\
\hline Ejaculation & \multicolumn{2}{|c|}{$81 \%$} & \multicolumn{2}{|c|}{$47 \%$} & \multicolumn{2}{|c|}{$75 \%$} & \multicolumn{2}{|c|}{$50 \%$} \\
\hline Mount latency* & 7.5 & 2.81 & 4.1 & 1.98 & 3.0 & 2.71 & 2.4 & 0.83 \\
\hline Intromission latency* & 8.0 & 2.78 & 15.0 & $2.87 \dagger$ & 6.6 & 3.92 & 11.1 & 4.87 \\
\hline Ejaculation latency* & 11.9 & 2.54 & 22.3 & $2.51 \S$ & 5.8 & 1.13 & 11.8 & $1.19 \dagger$ \\
\hline Number of mounts & 12.9 & 2.42 & 24.8 & $4.05 \dagger$ & 8.2 & 2.01 & 17.5 & $3.20 \dagger$ \\
\hline Number of intromissions & 4.2 & 0.70 & 3.0 & 0.71 & 3.0 & 1.08 & 2.2 & 0.60 \\
\hline Number of ejaculations & 1.4 & 0.20 & 0.7 & $0.23 \dagger$ & 0.9 & 0.44 & 0.9 & 0.48 \\
\hline Intromission ratio & 0.27 & 0.04 & 0.12 & $0.03 \dagger$ & 0.22 & 0.02 & 0.13 & $0.02 *$ \\
\hline
\end{tabular}

* Only animals that displayed the behavior. Latencies are in minutes. Significantly different from LRs as determined by $t$ test, ${ }^{+} p<.05,{ }^{*} p<.01$. 
The facility by which vaginal penetration is achieved depends on the activity of the striated penile muscles and adequate erection (Meisel \& Sachs, 1994; Sachs, 1983). Some data make it possible to propose deficiencies in both of these activities in the HRs. Stimulation of postsynaptic dopamine receptors by large doses of apomorphine has repeatedly been found to inhibit penile reflexes ex copula, including erections and flips (Benassi-Benelli, Ferrari, \& Quarantotti, 1979; Pehek, Thompson, Eaton, Bazzett, \& Hull, 1988), both of which are essential for the achievement of intromission (Sachs, 1983). Thus, since the HRs presumably have a heightened activity in dopaminergic systems, deficient penile reflexes might explain the fact that their intromission behavior is less efficient than that of the LRs. If the increased number of mounts in the HRs is a consequence of impaired intromission behavior, then there is no evidence whatsoever that these animals have a stronger sexual motivation than the LRs.

The difference in ambulatory activity between HRs and LRs is observed only in a novel environment. Thus, when these animals are retested without drug treatment, no difference is found (Piazza et al., 1989). The fact that, in the second experiment, locomotor response to amphetamine was greater for HRs than for LRs shows that these animals are indeed more sensitive to the locomotorstimulating effects of this drug. Nevertheless, sexual behavior responded (or failed to respond) in the same way in both groups. Because the two tests were not run at the same time, statistical comparisons cannot be made, but it seems that the mount latency was, at most, only modestly reduced after treatment with amphetamine. If anything, it appears to be more reduced in LRs than in HRs. In any case, it is safe to conclude that sexual behavior is not more facilitated in HRs than in LRs.

It could be argued that the impaired sexual behavior observed in the HRs in both experiments was a consequence of dopaminergic activity that was too elevated. Large doses of apomorphine have been shown to reduce sexual behavior, presumably because of the activation of incompatible behaviors (Malmnäs, 1973). Data from the second experiment, however, contradict such a proposal. If dopaminergic activity was too intense, and had thereby activated behaviors incompatible with copulation, this would have been intensified in the second experiment. This did not occur, because sexual behavior was not impaired by amphetamine. Furthermore, we have previously shown that doses of amphetamine that enhance such exploratory behaviors as sniffing or rearing in fact reduce mount and intromission latencies in naive animals ( $̊$ gmo \& Picker, 1990).

The present data raise questions of whether the distinction of HR-LR has any implication for sexual behavior. The data reviewed in the introduction show that HRs release more dopamine in the accumbens and are more sensitive to the effects of dopaminergic stimulants on ambulatory activity and self-administration. However, this does not necessarily mean that they are more sensitive to all actions of dopamine. In fact, it has recently been reported that there is no difference between HRs and LRs with regard to the strength of amphetamine-induced conditioned place preference (Erb \& Parker, 1994). Although HRs show enhanced ambulatory activity in a novel environment, another spontaneous behavior, sex, is of rather reduced intensity in these animals, both when untreated and after administration of amphetamine. It was recently reported that ethanol consumption was similar in HRs and LRs, showing that sensitivity to that reward is independent of reactions to novelty (Samson \& Chappelle, 1995). Thus, the distinction between HRs and LRs may have limited predictive value.

There was no difference between HRs and LRs at the second and third sexual behavior tests. This shows that whatever the exact cause of the difference at the first test might be, it is related to novelty and/or lack of sexual experience. In this context, it may be important to note that we did not directly determine dopamine release in our animals. There is, then, no neurochemical evidence that our HRs did, indeed, release more dopamine in response to novelty or after treatment with amphetamine than did LRs. However, since enhanced release has been found in several studies where HRs and LRs were obtained in the same way as ours were (Bradberry et al., 1991; Hooks et al., 1992; Hooks et al., 1991; Piazza, Rouge-Pont, et al., 1991), we did not feel any need to obtain neurochemical data. If differences in dopamine release are not reliable over studies, then the basic logic behind the distinction between HRs and LRs would be weak, and the behavioral interest of this distinction would be of questionable importance. It may also be observed that differences in transmitters other than dopamine could account for the differences observed in the present study.

It could also be argued that separation of the animals by the median is inadequate, because animals just above and just below this value are quite similar. Although this certainly is true, separation by the median is the way that HRs and LRs are obtained in most studies (see Methods for references). As mentioned in the introduction, important differences have been found between HRs and LRs thus obtained. Present data confirm that this distinction has behavioral consequences. Furthermore, if we compare the sexual behavior shown by the animals in the lowest quartile in the first test with that of those in the highest quartile, essentially the same differences as those obtained after separation by the median are observed (data not shown). This reinforces the notion that the latter procedure is valid.

To summarize, present data show that the main difference between HRs and LRs with regard to sexual behavior is a lower intromission ratio in the former. The other behavioral differences observed are most likely due to this fact. Further studies are needed before a role for dopamine in these effects can be firmly established.

\section{REFERENCES}

ÅgMO, A., \& FERnÁNDEZ, H. (1989). Dopamine and sexual behavior in the male rat: A reevaluation. Journal of Neural Transmission, 77, 21-37. Ágmo, A., \& Picker, Z. (1990). Catecholamines and the initiation of 
sexual behavior in male rats without sexual experience. Pharmacology, Biochemistry \& Behavior, 35, 327-334.

Ågmo, A., \& Villalpando, A. (1995). Central nervous stimulants facilitate sexual behavior in male rats with medial prefrontal cortex lesions. Brain Research, 696, 187-193.

Benassi-Benelli, A., Ferrari, F., \& Quarantotti, B. (1979). Penile erection induced by apomorphine and $N$ - $n$-propyl-norapomorphine in rats. Archives Internationales de Phamacodynamie et de Therapie, 242, 241-247.

Berlyne, D. E., KoeniG, D. V., \& Hirota, T. (1966). Novelty, arousal and the reinforcement of diversive exploration in the rat. Journal of Comparative \& Physiological Psychology, 62, 222-226.

Bradberry, C. W., Gruen, R. J., Berridge, C. W., \& Roth, R. H. (1991). Individual differences in behavioral measures: Correlation with nucleus accumbens dopamine measured by microdialysis. Pharmacology, Biochemistry \& Behavior, 39, 877-882.

Clark, J. T. (1993). Component analysis of male sexual behavior. In P. M. Conn (Ed.), Methods in neurosciences (Vol. 14, pp. 32-53). New York: Academic Press.

Contreras, J. L., \& $\AA$ Gmo, A. (1993). Sensory control of the male rat's copulatory thrusting patterns. Behavioral \& Neural Biology, 60 234-240.

Damsma, G., Pfaus, J. G., Wenkstern, D., Phillips, A. G., \& Fibiger, H. C. (1992). Sexual behavior increases dopamine transmission in the nucleus accumbens and striatum of male rats: Comparison with novelty and locomotion. Behavioral Neuroscience, 106, 181-191.

Davidson, J. M. (1980). The psychobiology of sexual experience. In J. M. Davidson \& R. J. Davidson (Eds.), The psychobiology of consciousness (pp. 271-332). New York: Plenum.

Deroche, V., Piazza, P. V., Le Moal, M., \& Simon, H. (1993). Individual differences in the psychomotor effects of morphine are predicted by reactivity to novelty and influenced by corticosterone secretion. Brain Research, 623, 341-344.

ERB, S. M., \& PARKER, L. A. (1994). Individual differences in noveltyinduced activity do not predict strength of amphetamine-induced place conditioning. Pharmacology, Biochemistry \& Behavior, 48, $581-586$

EveritT, B. J. (1990). Sexual motivation: A neural and behavioral analysis of the mechanisms underlying appetitive and copulatory responses of male rats. Neuroscience \& Biobehavioral Reviews, 14, 217-232

EXNER, M., \& ClARK, D. (1993). Behavior in a novel environment predicts responsiveness to $d$-amphetamine in the rat: A multivariate approach. Behavioural Pharmacology, 4, 47-56.

Hooks, M. S., Colvin, A. C., Juncos, J. L., \& Justice JR., J. B. (1992). Individual differences in basal and cocaine-stimulated extracellular dopamine in the nucleus accumbens using quantitative microdialysis. Brain Research, 587, 306-312.

Hooks, M. S., Jones, G. H., Smith, A. D., Neill, D. B., \& Justice JR., J. B. (1991). Response to novelty predicts the locomotor and nucleus accumbens dopamine response to cocaine. Synapse, 9, 121-128

Hooks, M. S., \& Kalivas, P. W. (1994). Involvement of dopamine and excitatory amino acid transmission in novelty-induced motor activity. Journal of Pharmacology \& Experimental Therapeutics, 269, 976-988.

Hooks, M.S., \& Kalivas, P. W. (1995). The role of the mesoaccumbenspallidal circuitry in novelty-induced behavioral activation. Neuroscience, 64, 587-597.

Hull, E. M., Du, J., Lorrain, D. S., \& Matuszewich, L. (1995). Extracellular dopamine in the medial preoptic area: Implications for sexual motivation and hormonal control of copulation. Journal of Neuroscience, 15, 7465-7471.

Hull, E. M., Weber, M. S., Eaton, R. C., Dua, R., Markowski, V. P., LuMLeY, L., \& Moses, J. (1991). Dopamine receptors in the ventral tegmental area affect motor, but not motivational or reflexive, components of copulation in male rats. Brain Research, 554, 72-76.

Louilot, A., Gonzalez-Mora, J. L., Guadalupe, T., \& Mas, M. (1991). Sex-related olfactory stimuli induce a selective increase in dopamine release in the nucleus accumbens of male rats: A voltammetric study. Brain Research, 553, 313-317.

Malmnäs, C. O. (1973). Monaminergic influences on testosteroneactivated copulatory behavior in the castrated male rat. Acta Physiologica Scandinavica, 395(Suppl.), 1-129.

MaLmNÄs, C. O. (1977). Dopaminergic reversal of the decline after castration of rat copulatory behavior. Journal of Endocrinology, 73, 187-188.

MeISEL, R. L., \& SACHS, B. D. (1994). The physiology of male sexual behavior. In E. Knobil \& J. D. Neill (Eds.), The physiology of reproduction (2nd ed., Vol. 2, pp. 3-105). New York: Raven.

Mitchell, J. B., \& Gratton, A. (1991). Opioid modulation and sensitization of dopamine release elicited by sexually relevant stimuli: A high speed chronoamperometric study in freely behaving rats. Brain Research, 551, 20-27.

Paredes, R. G., Holmes, G. M., Sachs, B. D., \& Ågmo, A. (1993). Electromyographic activity of rat ischiocavernosus muscles during copulation after treatment with a GABA transaminase inhibitor. $B e$ havioral \& Neural Biology, 60, 118-122.

Pehek, E. A., Thompson, J. T., Eaton, R. C., Bazzett, T. J., \& Hull, E. M. (1988). Apomorphine and haloperidol, but not domperidone, affect penile reflexes in rats. Pharmacology, Biochemistry \& Behavior, 31, 201-208.

Pfaus, J. G., Damsma, G., Nomikos, G. G., Wenkstern, D. G., Blaha, C. D., Phillips, A. G., \& Fibiger, H. C. (1990). Sexual behavior enhances central dopamine transmission in the male rat. Brain $R e$ search, 530, 345-348.

Piazza, P. V., Deminière, J. M., Maccari, S., Le Moal, M., \& SIMON, H. (1989). Factors that predict individual vulnerability to amphetamine self-administration. Science, 245, 1511-1513.

Piazza, P. V., Deminière, J. M., Maccari, S., Mormede, P., Le Moal, M., \& Simon, H. (1990). Individual reactivity to novelty predicts probability of amphetamine self-administration. Behavioral Pharmacology, 1, 339-345.

Piazza, P. V., Maccari, S., Deminière, J. M., Le Moal, M., MorMEDE, P., \& SimON, H. (1991). Corticosterone levels determine individual vulnerability to amphetamine self-administration. Proceedings of the National Academy of Sciences, 88, 2088-2092.

Piazza, P. V., Rouge-Pont, F., Deminière, J. M., Kharoubi, M., LE MOAL, M., \& SIMON, H. (1991). Dopaminergic activity is reduced in the prefrontal cortex and increases in the nucleus accumbens of rats predisposed to develop amphetamine self-administration. Brain Research, 567, 169-174.

Pierce, R. C., Crawford, C. A., Nonneman, A. J., Mattingly, B. A., \& BARDO, M. T. (1990). Effect of forebrain dopamine depletion on novelty-induced place preference behavior in rats. Pharmacology, Biochemistry \& Behavior, 36, 321-325.

SACHS, B. D. (1983). Potency and fertility: Hormonal and mechanical causes and effects of penile actions in rats. In J. Balthazart, E. Pröve, \& R. Gilles (Eds.), Hormones and behaviour in higher vertebrates (pp. 86-110). Berlin: Springer-Verlag.

Samson, H. H., \& Chappelle, A. M. (1995). Home-cage ethanol consumption and motor activity: Lack of relation to either initial activity or amphetamine-induced locomotion. Alcohol, 12, 37-42.

Scaletta, L. L., \& Hull, E. M. (1990). Systemic or intracranial apomorphine increases copulation in long-term castrated male rats. Pharmacology, Biochemistry \& Behavior, 37, 471-475.

SiLls, T. L., \& VACCARINo, F. J. (1994). Individual differences in sugar intake predict the locomotor response to acute and repeated amphetamine administration. Psychopharmacology, 116, 1-8.

Wenkstern, D., Pfaus, J. G., \& Fibiger, H. C. (1993). Dopamine transmission increases in the nucleus accumbens of male rats during their first exposure to sexually receptive female rats. Brain Research, $618,41-46$

(Manuscript received September 27, 1996; revision accepted for publication January $28,1997$. ) 